\title{
A virtual care program for outpatients diagnosed with COVID-19: a feasibility study
}

\author{
Philip W. Lam BScPhm MD, Prateek Sehgal MD, Nisha Andany MD MPH, Samira Mubareka MD, \\ Andrew E. Simor MD, Omar Ozaldin MSc, Jerome A. Leis MD MSc, Nick Daneman MD MSc, \\ Adrienne K. Chan MD MPH
}

\section{Abstract}

Background: In patients who are discharged home to self-isolate while coronavirus disease 2019 (COVID-19) test results are pending, there is no formal method for physician assessments or counselling to occur if the result returns positive. Our aim was to develop and test the feasibility of a virtual care program for self-isolating outpatients diagnosed with COVID-19.

Methods: In preparation for this gap in health care, the COVID-19 Expansion to Outpatients (COVIDEO) program was developed at the Sunnybrook Health Sciences Centre, Toronto, Ontario, to provide ongoing care for outpatients diagnosed with COVID-19. As part of a feasibility study, we describe our experiences with the first 50 patients managed using this program from its inception (Mar. 1, 2020) until Mar. 27, 2020.

Results: All 50 people who tested positive for COVID-19 at the Sunnybrook Health Sciences Centre and were discharged home to self-isolation during the study period were assessed through the COVIDEO program. Thirty-two patients (64\%) were assessed via the Ontario Telemedicine Network virtual care platform, and the remainder by telephone. The median time from viral swab collection to first COVIDEO program assessment was 2 (interquartile range [IQR] 1-2) days. Among the 26 patients for whom further follow-up care through the COVIDEO program was discontinued by the end of March 2020, the median duration of virtual care was 12.5 (IQR 8.75-16) days. During the study period, 6 patients required transfer to hospital for assessment, of whom 4 required admission.

Interpretation: We have shown that a virtual care program can be used in the management of outpatients diagnosed with COVID-19. Further studies evaluating its sustainability and impact on health outcomes are underway.

\footnotetext{
7 n December 2019, an outbreak of acute respiratory illness secondary to a novel coronavirus (SARS-CoV-2) originated in Wuhan, China. As of May 13, 2020, coronavirus disease 2019 (COVID-19) has caused a global pandemic resulting in more than 4170000 cases and over 287000 deaths. ${ }^{1}$ This pandemic has placed immense, and in some cases overwhelming, strain on health care systems around the world.

Most people with COVID-19 present initially with a mild illness and do not require hospital admission. ${ }^{2}$ These patients are generally discharged home to self-isolation as a means of reducing the burden on the health care system and limiting spread of COVID-19 to other, vulnerable patients and staff in hospital. ${ }^{3}$ We expected, however, that, owing to self-isolation requirements, there would be no formal method for physician assessments or counselling to occur in outpatients whose tests subsequently return as positive for COVID-19. The aim of our study was to develop and test the feasibility of a virtual care program for physician assessment and follow-up of outpatients with COVID-19 in self-isolation.
}

\section{Methods}

\section{Design and setting}

Sunnybrook Health Sciences Centre is a 627-bed academic tertiary care centre located in Toronto, Ontario. Patients who present to our institution with suspected COVID-19 infection and mild symptoms are sent home to self-isolate while test results are pending. Institutional testing criteria for COVID19 have evolved throughout the pandemic. Early in the pandemic, criteria for testing focused on patients with respiratory symptoms and a compatible history of travel to a high-risk country within the preceding 14 days. With ongoing local

\section{Competing interests: None declared.}

This article has been peer reviewed.

Correspondence to: Philip Lam, philip.lam@sunnybrook.ca CMAJ Open 2020. DOI:10.9778/cmajo.20200069 
community transmission becoming apparent in March 2020, the testing criteria became focused on those at highest risk for transmission to large groups of people (e.g., health care workers, people living in long-term care facilities), those sent for testing by Toronto Public Health and those most vulnerable to experiencing complications of infection (e.g., patients with cancer and those undergoing hemodialysis). To facilitate safe and efficient COVID-19 testing, an assessment centre separate from the hospital's Emergency Department was launched on Mar. 17, 2020.

\section{Participants}

All adult (age $\geq 18 \mathrm{yr}$ ) patients who were assessed at our institution, tested positive for COVID-19 and were discharged home to self-isolation from Mar. 1 to Mar. 27, 2020 were included in this study.

\section{Program development}

In anticipation of the gaps in care for outpatients diagnosed with COVID-19, the COVID-19 Expansion to Outpatients (COVIDEO) program was developed through an intradisciplinary collaboration between Microbiology, Infectious Diseases, and Infection Prevention and Control at Sunnybrook
Health Sciences Centre, and Toronto Public Health. The intradisciplinary team included all members of the Infectious Diseases Division, and smaller roles were played by infection prevention and control leadership and practitioners, as well as microbiology leadership and reporting technicians. The program was developed from Feb. 17 to Feb. 28, 2020, during which time the aforementioned groups met to create the workflow and standardized assessment forms.

\section{Program processes and procedure}

Figure 1 outlines the workflow of the COVIDEO program once a new positive COVID-19 test result in an outpatient is identified. The Infection Prevention and Control Team communicates the positive test result and patient information by secure email to the infectious diseases physician assigned to the COVIDEO program. One infectious diseases physician is assigned to the COVIDEO program each week; the program is maintained through a rotating weekly schedule among 5 infectious diseases physicians.

Once the patient information is received, the infectious diseases physician contacts the patient by telephone to inform him or her of the positive result and to arrange a virtual care assessment using the Ontario Telemedicine Network virtual

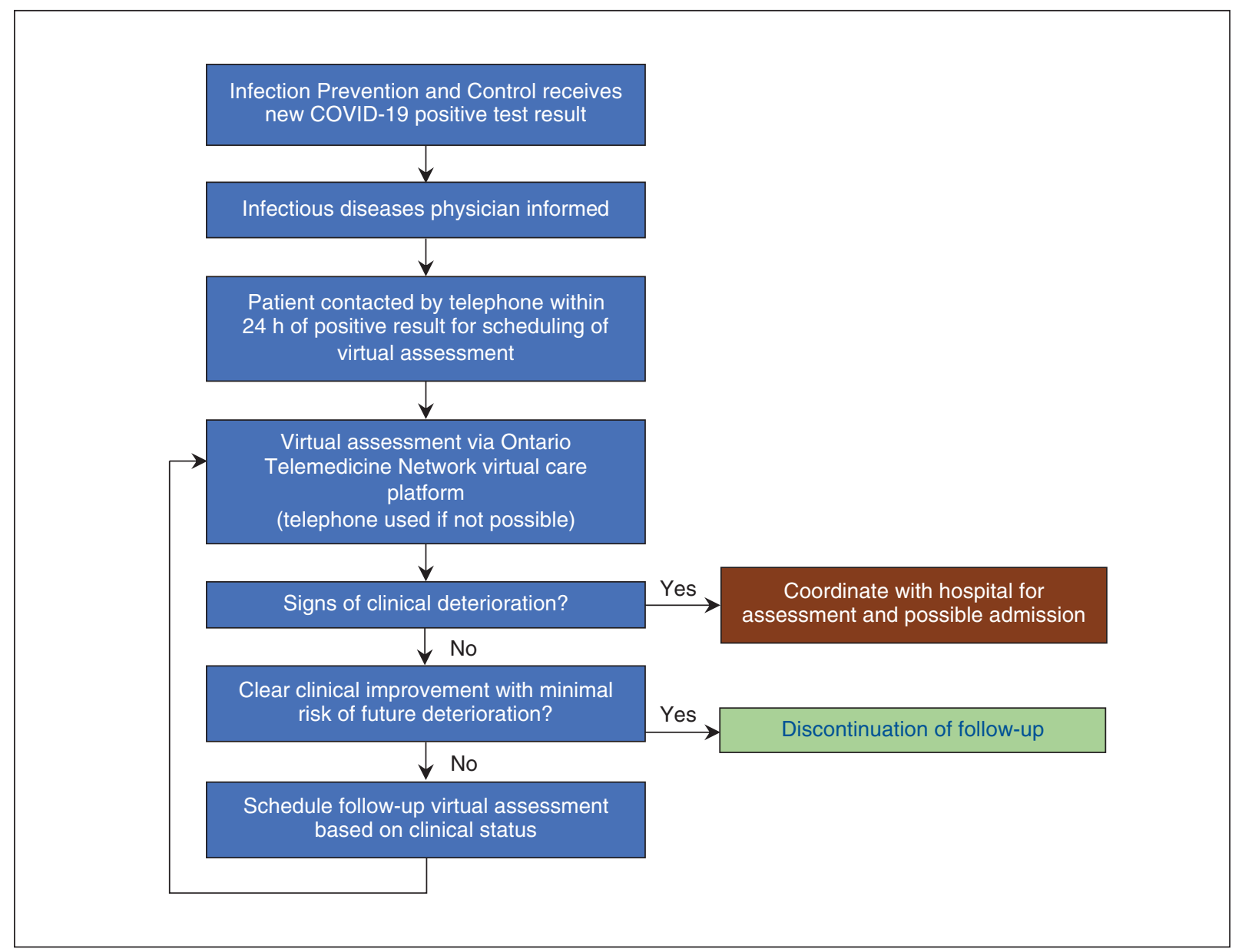

Figure 1: Coronavirus disease 2019 (COVID-19) Expansion to Outpatients program process map for the management of new cases of COVID-19 in outpatients who are in self-isolation. 
care platform, a government-funded, nonprofit organization that currently serves as the primary platform for virtual care services in Ontario. ${ }^{4}$ Patients who are unable to use or decline the use of the Ontario Telemedicine Network platform are assessed by telephone.

Standardized forms for initial and follow-up assessments were developed to facilitate each virtual appointment (Appendix 1, available at www.cmajopen.ca/content/8/2/E407/suppl/ DC1) and revised iteratively based on the accumulation of clinical experience through this program. The initial assessment consists of a detailed review of the patient's travel history, exposure to anyone infected with COVID-19, comorbidities and current symptoms. Questions pertaining to the patient's breathing, ability to walk and exercise are also included. The follow-up assessments are focused on changes in patient-reported symptoms. If a household contact of the patient being assessed is also symptomatic, the infectious diseases physician forwards the patient's information through secure email to the Toronto Public Health Communicable Diseases Liaison Unit so that further contact tracing can be performed. During these assessments, patients are counselled on the nature of COVID-19, the spectrum of illness it can produce, the complications that may occur and worrisome symptoms (such as progressive shortness of breath) that should prompt the patient to seek medical attention. Patients who experience any worrisome symptoms are instructed to activate a hospital pager number that directly notifies the COVIDEO infectious diseases physician.

At the end of each assessment, the infectious diseases physician, based on clinical judgment, determines whether the patient's clinical status is stable, improving or worsening. In patients whose clinical status is deemed stable, follow-up assessments are conducted by the infectious diseases physician on a weekly basis, at a minimum, until clearance by Toronto Public Health. In the intervening period, patients are also contacted by telephone by their Toronto Public Health case worker, who notifies the infectious diseases physician if there are any concerns. Patients who are deemed to require more frequent follow-up are contacted up to twice a day; the exact schedule of follow-up is at the discretion of the infectious diseases physician based on clinical judgment and guided by each patient's risk factors for severe infection.

In the event of clinical deterioration requiring hospital transfer, the infectious diseases physician coordinates with the Infection Prevention and Control Team and the hospital Emergency Department triage or COVID-19 ward to ensure that transportation and admission to hospital is completed in a controlled and safe manner. Strategies used to ensure this include having a family member transport the patient to hospital (if possible), having the patient sit in the opposite corner of the vehicle, having the patient and transporter wear masks, ensuring the windows are open during transportation, ensuring the patient performs hand hygiene on entering the hospital, and ensuring a health care worker with appropriate personal protective equipment can meet the patient at a designated hospital entrance and escort him or her directly to an isolation room.
The decision to discontinue any further COVIDEO follow-up is based on the infectious diseases physician's determination that there has been clear clinical improvement and minimal risk of future deterioration.

The COVIDEO program was launched on Mar. 1, 2020. For each patient enrolled in the COVIDEO program, success was defined by completion of 1 initial assessment and at least 1 follow-up assessment. For the program as a whole, we focused on attaining at least $90 \%$ enrolment for all outpatients diagnosed with COVID-19.

\section{Statistical analysis}

We conducted a descriptive analysis of patient demographic characteristics, travel history, comorbidities, symptom profile and investigations.

\section{Ethics approval}

Research ethics board review was not required because the study met the Sunnybrook Research Institute criteria for exemption based on a standardized process for confirming that the project focused on improvement in quality of care and not human subject research.

\section{Results}

From Mar. 1 to Mar. 27, 2020, 50 people assessed at Sunnybrook Health Sciences Centre for possible COVID-19 and discharged home to self-isolation tested positive for COVID-19. During the same period, 13 people under investigation were admitted to hospital and tested positive for COVID-19. All 50 outpatients were assessed successfully by an infectious diseases physician using the COVIDEO program. Of the 50, 32 (64\%) were assessed via the Ontario Telemedicine Network virtual care platform; the remainder were assessed by telephone. The median age of the patients was 44 (interquartile range [IQR] 30.5-59.5) years, and $26(52 \%)$ had travelled in the preceding 14 days (Table 1). The most common presenting symptoms were cough $(34[68 \%])$, fatigue $(32[64 \%])$ and headache $(22[44 \%])$ (Table 1$)$. The case count during the study period stratified by travel history is illustrated in Figure 2 .

The median time from viral swab collection to first COVIDEO assessment was 2 (IQR 1-2) days. The maximum time from viral swab collection to first COVIDEO assessment was 14 days; this particular patient was diagnosed with COVID-19 during international travel and presented to our institution 12 days after initial viral swab collection. Among the 26 patients in whom further COVIDEO follow-up was discontinued, the median duration of virtual care was 12.5 (IQR 8.75-16) days. As of Apr. 4, 2020, 23 (46\%) of the 50 patients continued to be followed by the COVIDEO program. The care trajectory for each of the 50 patients assessed is summarized in Figure 3.

Six patients (12\%) showed a change in clinical status requiring transfer to hospital; only 1 of these transfers occurred without notification and coordination by the COVIDEO program. Four of the 6 patients were admitted to hospital. The median age of these 6 patients was 61 (IQR 56.5-64.75) years, and 


\section{Table 1 (part 1 of 2): Characteristics of the first 50 outpatients diagnosed with COVID-19 managed with the COVID-19 Expansion to Outpatients virtual care program}

\begin{tabular}{lc} 
Characteristic & No. (\%) of patients \\
\hline Demographic & \\
\hline Age, yr, median (IQR) & $44(30.5-59.5)$ \\
\hline Male sex & $33(66)$ \\
$\begin{array}{l}\text { Days from symptom onset to health care } \\
\text { assessment, median (IQR) }\end{array}$ & $3(1.5-5.0)$
\end{tabular}

assessment, median (IQR)

Travel within $14 \mathrm{~d}$ of symptom onset $\dagger \quad 26(52)$

Austria 3

Canada (within country) 2

Cayman Islands 1

Colombia 1

Dominican Republic 1

Egypt 2

England 1

Germany 1

Iran 3

Japan 1

Peru 2

Switzerland 1

United Arab Emirates 2

United States 10

Health care worker 17 (34)

Community acquisition 9

Travel within $14 \mathrm{~d}$ of symptom onset 8

\section{Comorbidities}

\begin{tabular}{|lc|}
\hline Asthma & $3(6)$ \\
\hline Cardiac disease & $1(2)$ \\
\hline Chronic hematologic disease & $2(4)$ \\
\hline Chronic kidney disease & $2(4)$ \\
\hline Chronic lung disease & $1(2)$ \\
\hline Chronic neurologic disease & $1(2)$ \\
\hline Diabetes & $3(6)$ \\
\hline HIV infection & $0(0)$ \\
\hline Hypertension & $11(22)$ \\
\hline Malignant disease & $5(10)$ \\
\hline Malnutrition & $0(0)$ \\
\hline Moderate to severe liver disease & $0(0)$ \\
\hline Obesity & $1(2)$ \\
\hline Rheumatic disorder & $1(2)$ \\
\hline Smoking & $5(10)$ \\
\hline
\end{tabular}

$5(83 \%)$ of the 6 were men. The median time from diagnosis of COVID-19 to hospital transfer was 4 (IQR 2.25-6.5) days. The reasons for transfer included changes in respiratory status (3 patients) and, in 1 case each, chest pain, relative hypotension
Table 1 (part 2 of 2): Characteristics of the first 50 outpatients diagnosed with COVID-19 managed with the COVID-19 Expansion to Outpatients virtual care program

Characteristic

No. $(\%)$ of patients*

Presenting symptoms

Abdominal pain $8(16)$

Adenopathy $0(0)$

Anorexia $21(42)$

Arthralgia $5(10)$

Chest pain $7(14)$

Chills/rigors $18(36)$

Confusion $1(2)$

Conjunctivitis $3(6)$

Cough $34(68)$

Depression and anxiety 9 (18)

Diarrhea $15(30)$

Dyspnea $10(20)$

Fatigue/malaise $32(64)$

Fever 19 (38)

Headache $22(44)$

Hemoptysis $0(0)$

Insomnia $13(26)$

Myalgia $21(42)$

Nausea and vomiting $10(20)$

Otalgia $5(10)$

Pharyngitis 19 (38)

Rash $0(0)$

Rhinorrhea $13(26)$

Sputum production 16 (32)

Wheezing 2 (4)

Investigations

Complete blood count $13(26)$

Leukocyte count $\times 10^{9} / \mathrm{L}$, median $\quad 4.9$

Lymphocyte count $\times 10 \%$, median 0.9

Chest radiography 15 (30)

Abnormal findings $\quad 11$

Bilateral infiltrates $\quad 7$

Note: COVID-19 = coronavirus disease 2019, IQR = interquartile range.

*Except where noted otherwise.

†Some patients had travelled to more than 1 country.

and need for isolation owing to ongoing hemodialysis. As of Apr. 4, 2020, 1 patient had been admitted to intensive care, and no patient had died.

\section{Interpretation}

We have shown the feasibility of a virtual care program using a collaborative approach in the management of outpatients 


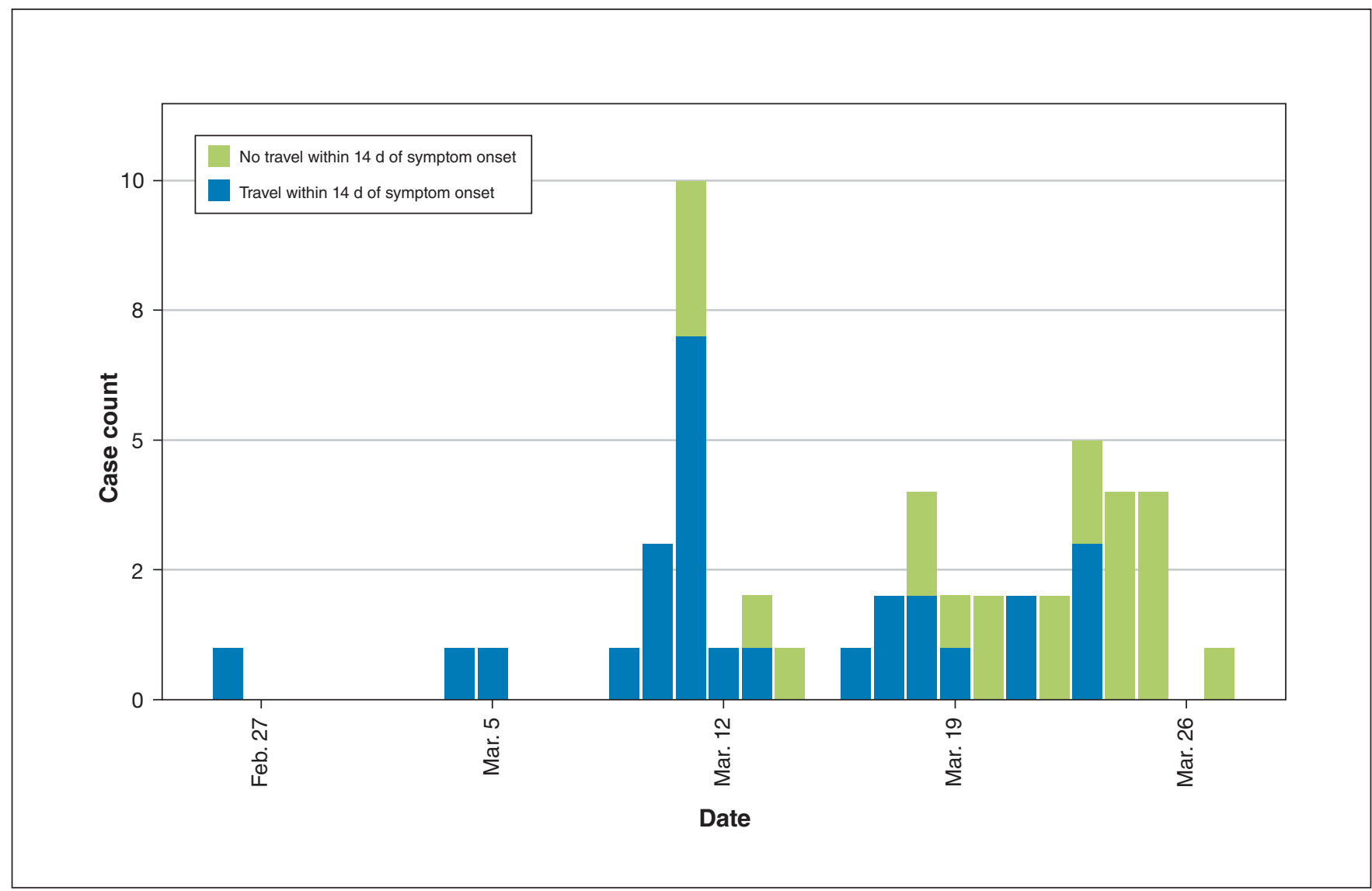

Figure 2: Case count for the 50 outpatients diagnosed with coronavirus disease 2019 (COVID-19) assessed through the COVID-19 Expansion to Outpatients program, stratified by travel history.

diagnosed with COVID-19. The use of virtual care in medicine has become an attractive strategy to minimize unnecessary patient travel to health care institutions for medical care and has a unique application in patients who require selfisolation. In addition to the care provided to the patient, potential benefits of a virtual care program include the ability for health care providers to plan for a safe and controlled hospital transfer for those showing signs of clinical deterioration, and the ability to reduce further community spread by informing public health of any household contacts with symptoms.

We identified several challenges in implementing the COVIDEO program. First, a substantial proportion of assessments were completed by telephone as opposed to through the Ontario Telemedicine Network platform, as intended. Contributing factors likely included a shift toward older people (owing to changes in testing criteria), who may not be as comfortable using such technology; the inability of family members or caregivers to assist owing to self-isolation requirements; and patients' symptoms, which may push them to choose a simpler modality such as the telephone. Physicianrelated factors that presented challenges included the increased number of patients accrued over time combined with the administrative challenges of booking virtual visits, with some occurring on an impromptu basis. Physicians should ensure there is sufficient administrative support to facilitate a virtual care program. Likewise, patients should be counselled on the added benefits of video, such as the additional visual information available to the physician (e.g., increased work of breathing or cyanosis) and a greater therapeutic presence. ${ }^{5}$

We also encountered challenges in managing patients with ongoing dyspnea. Patients with COVID-19 with dyspnea are at higher risk for complications such as acute respiratory distress syndrome, ${ }^{6}$ and the ability to assess for hypoxemia is limited in patients under self-isolation at home. It is also unclear whether subjective scoring systems such as the Roth score ${ }^{7}$ and the Medical Research Council breathlessness scale $^{8}$ correlate well with hypoxemia in the context of COVID-19. To address this, we have procured portable oximeters, which are now delivered to the patient's home for twice-daily monitoring in those who report severe dyspnea or are considered to be at risk for severe infection because of age, pregnancy or comorbidities.

\section{Limitations}

There are several limitations to our virtual care program. Given the urgency of the pandemic and our desire to share our experiences with others quickly, we are unable to comment on the long-term sustainability of such a program. As the number of patients diagnosed with COVID-19 continues 
Research

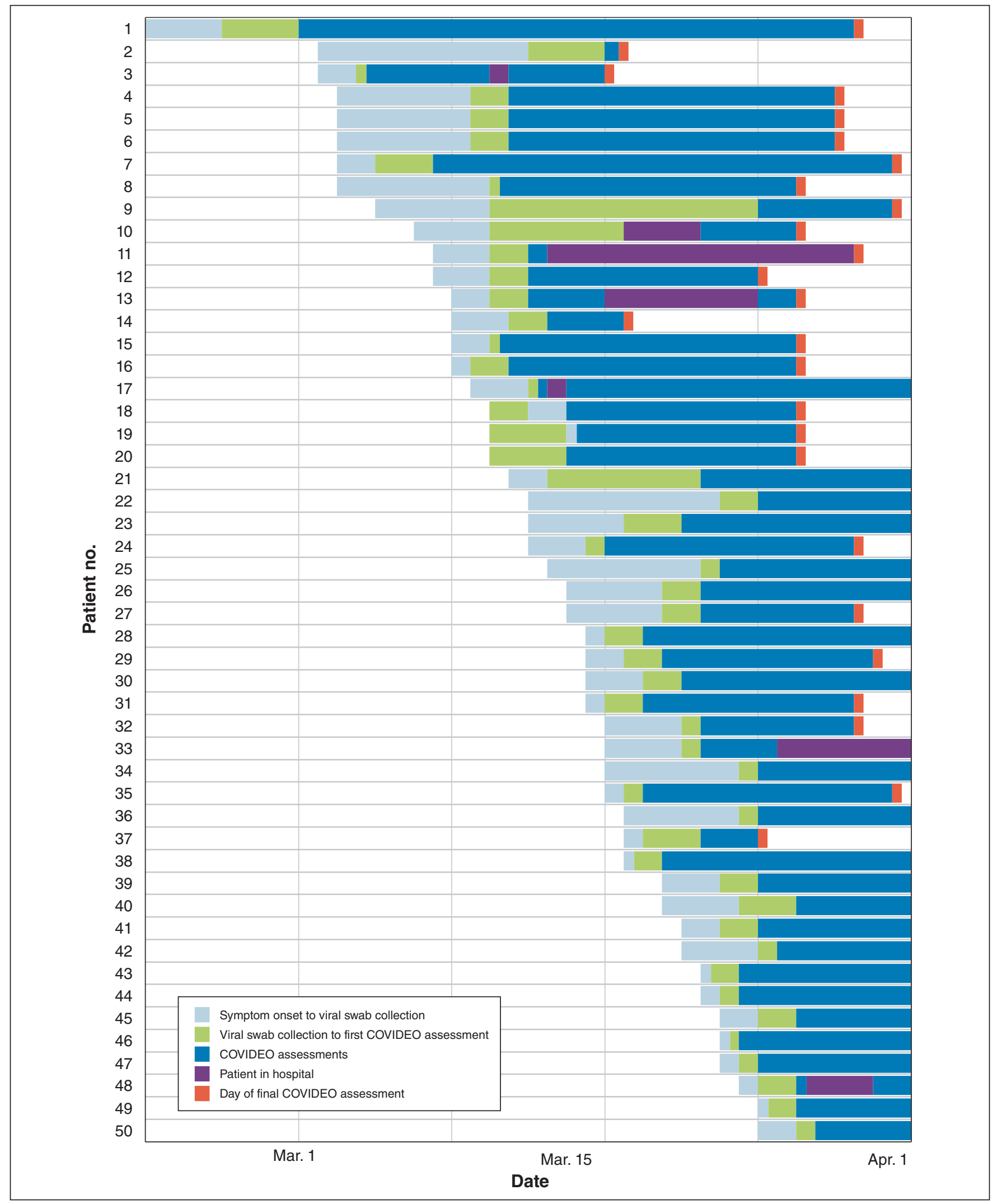

Figure 3: Care trajectory for the 50 outpatients diagnosed with coronavirus disease 2019 (COVID-19) assessed through the COVID-19 Expansion to Outpatients (COVIDEO) program up until Apr. 2, 2020. Dark blue bars that extend to Apr. 1 reflect patients who were still being followed actively by the COVIDEO program at the time of data collection. One patient (row 10) was admitted to hospital during the initial presentation and then discharged home before the diagnosis of COVID-19 was confirmed. Note: day of final COVIDEO assessment = discontinuation of further follow-up. 
to increase, the COVIDEO program will inevitably become more time- and labour-intensive. We think that the program could be sustainable, scaled and adapted for use by other primary care providers to manage outpatients diagnosed with COVID-19, potentially through a rotating roster of physicians or nurse practitioners linked to assessment centres who are willing to provide care for patients for the duration of illness. Importantly, for practising physicians and trainees who may not be able to assist in the direct care of people with confirmed or suspected COVID-19 owing to underlying comorbidities, this virtual care platform may provide them with an opportunity to contribute to COVID-19-related care in a safe yet effective way.

Owing to implementation of the COVIDEO program early in the pandemic to improve quality of care, no baseline or comparator data were available to evaluate the incremental impact of the program on outcomes. We plan to evaluate our intervention formally by conducting a controlled quasiexperimental study comparing outcomes of patients in our COVIDEO program to patients treated at other institutions in Ontario through linkage to administrative databases. We also plan to evaluate the impact of our program on the patient experience formally using qualitative methods.

Marginalized populations who may not have access to a telephone or the Internet, in particular those who are underhoused, may be challenging to manage. In these instances, it is important that clinicians familiarize themselves and forge relationships with local community outreach teams, transitional housing and shelter systems to bridge the gap in patient care.

Finally, the short time frame between viral swab collection and first assessment through the COVIDEO program was dependent on a rapid turnaround time for COVID-19 testing at our institution. Settings where reporting of COVID-19 test results is delayed may attenuate the benefits of early assessments in outpatients with COVID-19 in self-isolation.

\section{Conclusion}

It is difficult to predict the final toll of COVID-19, but it appears that virtual care may be a feasible way to manage outpatients who are self-isolating. We plan to evaluate the health impact of this virtual care program formally once we have recruited a sufficient number of patients into the program. In the meantime, our COVIDEO virtual care program may serve as a template for other institutions in providing care to outpatients diagnosed with COVID-19.

\section{References}

1. Coronavirus disease 2019 (COVID-19): situation report - 114. Geneva: World Health Organization; updated 2020 May 13. Available: https://www. who.int/docs/default-source/coronaviruse/situation-reports/20200513-covid-19 -sitrep-114.pdf?sfvrsn=17ebbbe 4 (accessed 2020 May 13).

2. Novel Coronavirus Pneumonia Emergency Response Epidemiology Team. The epidemiological characteristics of an outbreak of 2019 novel coronavirus diseases (COVID-19) in China [article in Chinese]. Zhonghua Liu Xing Bing Xue Za Zhi 2020;41:145-51.

3. Lin M, Beliavsky A, Katz K, et al. What can early Canadian experience screening for COVID-19 teach us about how to prepare for a pandemic? CMA7 2020;192:E314-8.

4. Brown EM. The Ontario Telemedicine Network: a case report. Telemed $7 \mathrm{E}$ Health 2013;19:373-6.

5. Greenhalgh T, Koh GCH, Car J. Covid-19: a remote assessment in primary care. $B M \mathcal{F} 2020 ; 368: \mathrm{m} 1182$.

6. Wu C, Chen X, Cai Y, et al. Risk factors associated with acute respiratory distress syndrome and death in patients with coronavirus disease 2019 pneumonia in Wuhan, China. 7AMA Intern Med 2020 Mar. 13. [Epub ahead of print]. doi: 10.1001/jamainternmed.2020.0994.

7. Chorin E, Padegimas A, Havakuk O, et al. Assessment of respiratory distress by the Roth score. Clin Cardiol 2016;39:636-9.

8. Stenton C. The MRC breathlessness scale. Occup Med (Lond) 2008;58:226-7.

Affiliations: Department of Medicine (Lam, Sehgal, Andany, Mubareka, Simor, Leis, Daneman, Chan), University of Toronto; Division of Infectious Diseases (Lam, Andany, Mubareka, Simor, Leis, Daneman, Chan), Sunnybrook Health Sciences Centre; Toronto Public Health (Ozaldin), Toronto, Ont.

Contributors: Nick Daneman and Adrienne Chan supervised the project. Philip Lam, Nisha Andany, Samira Mubareka, Jerome Leis, Nick Daneman and Adrienne Chan conceived the work. Philip Lam and Nick Daneman designed the work and analyzed the data. Nisha Andany, Adrienne Chan, Nick Daneman, Philip Lam and Prateek Sehgal acquired and interpreted the data. Philip Lam, Prateek Sehgal, Nick Daneman and Adrienne Chan drafted the manuscript, and Prateek Sehgal, Nisha Andany, Samira Mubareka, Andrew Simor, Omar Ozaldin, Jerome Leis, Nick Daneman and Adrienne Chan revised it critically for important intellectual content. All of the authors approved the final version to be published and agreed to be accountable for all aspects of the work.

Data sharing: Research ethics board review was not required because the study met our institutional criteria for exemption based on a standardized process for confirming that the project focused on improvement in quality of care and not human subject research. As such, approval for datasharing was not obtained; investigators may contact the corresponding author for further inquiries: Philip Lam, philip.lam@sunnybrook.ca.

Supplemental information: For reviewer comments and the original submission of this manuscript, please see www.cmajopen.ca/content/8/2/ E407/suppl/DC1. 